\title{
Article \\ Optimization of Acoustic Communication Links for a Swarm of AUVs: The COMET and NEMOSENS Examples
}

\author{
Camila M. G. Gussen ${ }^{1, *}$, Christophe Laot ${ }^{1}$, François-Xavier Socheleau ${ }^{1}$, Benoît Zerr ${ }^{2}$, Thomas Le Mézo ${ }^{2}$, \\ Raphaël Bourdon ${ }^{3}$ and Céline Le Berre ${ }^{3}$
}

1 IMT Atlantique Bretagne-Pays de la Loire, Lab-STICC, UMR CNRS 6285, 29238 Brest, France; christophe.laot@imt-atlantique.fr (C.L.); fx.socheleau@imt-atlantique.fr (F.-X.S.)

2 ENSTA Bretagne, Lab-STICC, UMR CNRS 6285, 29806 Brest, France; benoit.zerr@ensta-bretagne.fr (B.Z.); thomas.le_mezo@ensta-bretagne.org (T.L.M.)

3 RTSys, ZA de Kerloïc, Rue Jean-Noël Jégo, 56850 Caudan, France; rbourdon@rtsys.fr (R.B.); cleberre@rtsys.fr (C.L.B.)

* Correspondence: camila-maria.gabriel-gussen@imt-atlantique.fr

Citation: Gussen, C.M.G.; Laot, C.; Socheleau, F.-X.; Zerr, B.; Le Mézo, T.; Bourdon, R.; Le Berre, C.

Optimization of Acoustic Communication Links for a Swarm of AUVs: The COMET and NEMOSENS Examples. Appl. Sci. 2021, 11, 8200. https://doi.org/10.3390/app11178200

Academic Editor: Beatrice Tomasi

Received: 30 July 2021

Accepted: 1 September 2021

Published: 3 September 2021

Publisher's Note: MDPI stays neutral with regard to jurisdictional claims in published maps and institutional affiliations.

Copyright: (C) 2021 by the authors Licensee MDPI, Basel, Switzerland. This article is an open access article distributed under the terms and conditions of the Creative Commons Attribution (CC BY) license (https:/ / creativecommons.org/licenses/by/ $4.0 /$ )

\begin{abstract}
Underwater acoustic communication is a key point for performance improvement in an autonomous underwater vehicle (AUV) swarm. The communication process is essential for improving the AUVs localization accuracy for navigation and is a convenient way for sharing information among the AUVs in a network. The objective of this work, which was developed in the COMET and NEMOSENS projects, is to address the communication process required in a mobile underwater wireless network, with a focus on the proposal of an adaptive physical layer methodology. We discuss about the employed channel access method, the frame structure, and we propose the usage of an adaptive guard interval in order to ameliorate the network usage rate. We explain the physical layer aspect of the communication: the data processing at the transmitter and receiver side. In addition to that, we propose the usage of smart communications among AUVs. We design a method for adapting some physical layer parameters. The proposed approach relies only on the knowledge of the transmission geometry, and it optimizes the number of subcarriers and the cyclic-prefix length of the Orthogonal Frequency-Division Multiplexing (OFDM) system. The obtained results show a performance improvement in terms of bit-error rate when compared with the case of random parameters selection. These results corroborate the benefits of our adaptive parameters approach.
\end{abstract}

Keywords: underwater communication; underwater acoustic communication; autonomous underwater vehicle; AUV; swarm of AUVs; adaptive underwater communication; underwater OFDM system

\section{Introduction}

A swarm of autonomous underwater vehicles (AUVs) can be the key for a better exploration of the underwater environment. Many underwater applications can benefit from the usage of several underwater vehicles working together. Possible applications include seabed mapping, plume tracking [1], studies on fish migration [2], sea pollution monitoring, corrosion prevention in offshore installation of wind farms, undersea surveillance [3], among other civilian and military applications [4].

The main motivation for using many AUVs is that a single AUV might not be able to perform the same task as a swarm. As an example, the usage of a single underwater vehicle poses critical constraints for tracking plumes [1]. The main problem is the aliasing of spatial or temporal samples. For the cases of sensing small-scale features in the water, the AUV needs to perform high spatial sampling, which limits the survey coverage area (not enough space resolution). For the cases of dynamic features, such as hydrothermal vent plumes, the plume moves before the AUV survey is completed (lack of time resolution) [1]. Therefore, the usage of a set of coordinated AUVs can address all these aforementioned issues. 
Another benefit of a swarm of AUVs is that it can improve the localization accuracy for navigation purposes. In addition to that, a swarm enables the coverage of a wider area, and data acquisition with a better quality (without aliasing problems). The benefits go beyond, and include a better system reliability [4].

A swarm of AUVs is composed of several autonomous vehicles that act in a coordinated manner. They are designed to work together for accomplishing a pre-established common mission. In order to enable the collaboration among the distinct AUVs belonging to the same network, a reliable communication is mandatory [5].

The communication system is composed of several stages, including the medium access control and the physical layer. A possible way of improving the overall system performance is through the adaptation of some physical layer parameters. The adaptation of such parameters can be an efficient way of maintaining the quality-of-service in the highly time-variant acoustic channel.

The majority of the adaptive approaches available in the literature requires some knowledge of the channel, which are obtained after an initial information exchange between transmitter and receiver, i.e., they require a feedback link. As an example, in [6] their objective is to maximize the throughput, with the constraint of maintaining an average bit error rate (BER). In this case, the adaptive parameters of the OFDM system are the modulation and/or the power level of each subcarrier. For performing these adaptation, this approach utilizes a channel prediction of one travel time ahead. The work from [7] aims to maximize the transmission rate for a given transmission power. For achieving this objective, they propose a new performance metric for selecting the most convenient modulation and coding scheme. This pair of adaptive parameters belongs to a previously defined set of transmission modes. Another reference [8] proposes the usage of a sparse principal component analysis (SPCA) for the selection of modulation and coding parameters. Some recent works propose the usage of reinforcement learning $[9,10]$ and of machine learning (ML) [11] algorithms for adapting the modulation and coding parameters. The works from $[12,13]$ utilize a multi-user game theory approach for adapting the power level of each user in an OFDM system. All these aforementioned works relies on some kind of channel state information (CSI).

Although, for some underwater applications, this feedback link might not be available. A possibility is to utilize high-level environmental information for improving the system performance. The reference [14] proposes a blind adaptive modulation technique, which is based on machine learning. This approach depends on the accuracy of the considered propagation model.

A contribution of this paper is the presentation and discussion of all the procedures required to perform communications among a swarm of AUVs. A special focus is given to physical layer optimization. In addition to that, we propose a method for a smart use of the time-frequency resources with self-adaptation based on a limited knowledge of the environment for an orthogonal frequency-division multiplexing (OFDM) system (part of this work has been presented at the Underwater Acoustics Conference and Exhibition (UACE 2021)). In this new approach, the number of subcarriers and the cyclic prefix length are optimized. Moreover, we provide simulation results for showing the performance gain obtained in terms of BER with our method.

This work is part of COMET and NEMOSENS projects, which is supervised by RTSYS company and is funded by Region Bretagne and BPI France. The aim is to develop swarms of AUVs for distinct oceanographic missions. Our focus is to improve the communication among these vehicles, depending on the network topology. In such network, with no central hierarchy, each node is able to self-adapt its transmission parameters.

Knowing that the AUVs location and speed is periodically broadcasted, we can take advantage of this information. This channel geometry data can be used for adapting some transmission parameters, such as the number of subcarriers, cyclic prefix length, as well the guard interval. In this network, each AUV is going to adapt its parameters in order to improve the overall system performance. 
This paper is organized as follows. We start the paper by presenting the underwater vehicles to be used in a swarm in Section 2. In Section 3, we show the benefits that the usage of underwater acoustic communication brings to a swarm of AUVs. Section 4 presents the developed channel access method and the frame structure. The aspects regarding the physical layer are addressed in Section 5. This section contains the modem description, proposes a method for adapting some physical layer parameters, and provides simulation results. At the end, Section 6 shows our conclusions.

\section{Autonomous Underwater Vehicles}

Two distinct AUVs are considered in this work. The first one is COMET, which is shown in Figure 1. COMET can work in a swarm mode with up to 10 AUVs. The acoustic communication system enables them to have an accurate positioning information [15]. A precise underwater positioning is useful not only for swarm navigation, but also for field data collection. These surveyed data are associated with a more reliable ocean coordinates. This AUV can operate in depths up to $300 \mathrm{~m}$ and its battery can last up to $20 \mathrm{~h}$. COMET is around $2 \mathrm{~m}$ long and it weights less than $40 \mathrm{~kg}$.

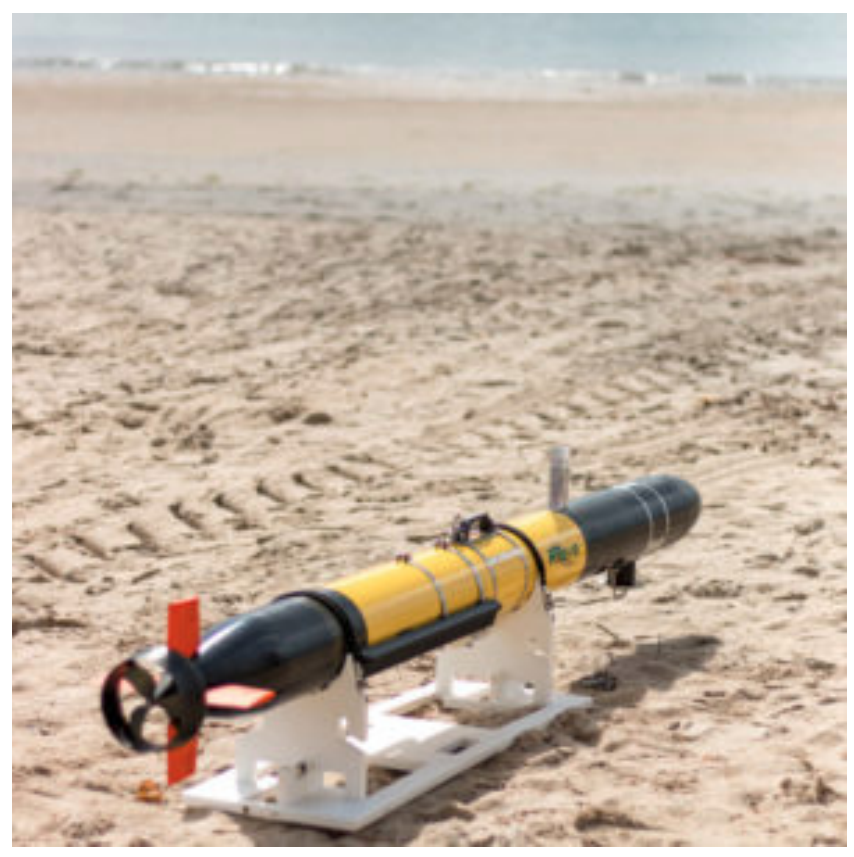

Figure 1. AUV COMET.

The second AUV is NemoSens, which is depicted in Figure 2. NemoSens is a micro AUV, and it is $90 \mathrm{~cm}$ long, has a diameter of $120 \mathrm{~mm}$, and weights less than $9 \mathrm{~kg}$. This micro AUV can operate in a swarm mode with up to seven vehicles, which extends the mission coverage [16]. NemoSens is able to perform missions up to a depth of $300 \mathrm{~m}$, with a battery autonomy up to $10 \mathrm{~h}$. Another differential feature of this AUV is its flexible system. With the open Linux architecture, it is possible to test and employ any other navigation algorithm. 


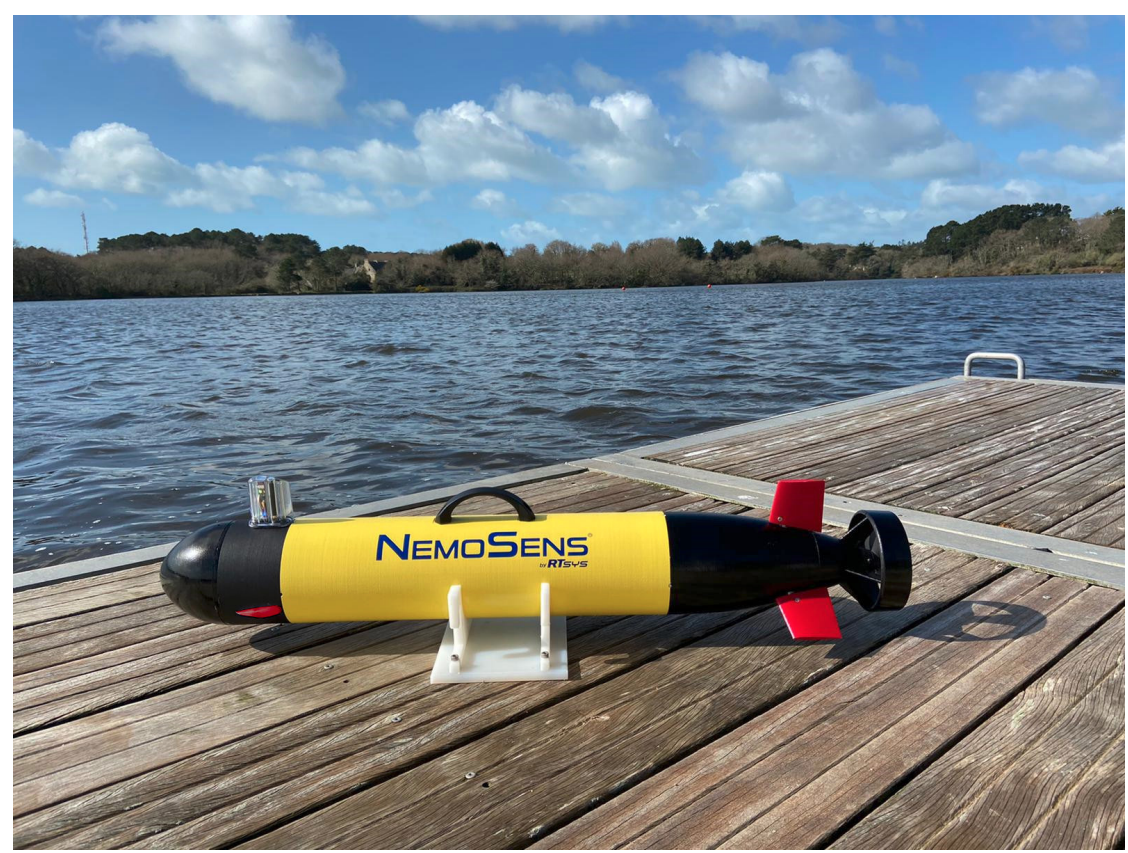

Figure 2. AUV NemoSens.

\section{Importance and Benefits of Underwater Communications}

Navigating in the underwater environment is a complex task [17]. The navigation system is also responsible for detecting underwater objects such as hazardous wreck, rocks, seafloor modifications, and other vehicles, in order to avoid collision. Both COMET and NemoSens AUVs utilizes the Inertial Navigation System (INS) aided by Doppler Velocity Log (DVL), and when available, the Global Positioning System (GPS) or Long Baseline (LBL) $[15,16]$. In one configuration of NemoSens, the DVL can be suppressed, reducing drastically the AUV cost. In this case, the acoustic communications among AUVs plays a major role in maintaining an accurate navigation.

When the AUVs operate together in a swarm mode, the navigation algorithm can be improved with the aid of the signal received from the other vehicles. This means that the navigation can be adapted according to information received. In addition to that, there is the need of an algorithm for coordinating the swarm navigation. As there is no central node in this network, each AUV can use the knowledge of the network topology, which is obtained in the process of information exchange.

Another important improvement that can be achieved thanks to underwater communication is with respect to localization. When the AUV is at the surface, the localization system utilizes the GPS. Once the AUV dives, the vehicle only gets the measurements acquired by its subsea sensors. These measurements have inherent uncertainties, which are represented by the boxes in Figure 3, part 1. When the AUVs operate in a swarm mode, these vehicles might take profit of the information exchange among them for improving localization. While in hearing mode, the AUV can measure the time of arrival of each signal (see Figure 3, part 2). With this information, the AUV can compute the location range that the transmitting vehicle is situated (see Figure 3, part 3). Using the knowledge of the transmitter position box (that was transmitted in the message), the receiver node can improve the localization accuracy of the transmitter AUV, as shown in Figure 3, part 4. These continuous information exchanges among users enables the contraction of position boxes, increasing the localization accuracy [18].

A benefit of having a more precise position is a better coverage [18]. Figure 4, depicts the area coverage for the case when the AUVs exchange information and for the case the vehicles do not communicate. In this figure, the red color corresponds to the unexplored areas, and the green intensity designates the amount of scans that were performed. 
From this figure, one can observe the improvement on the area coverage when the AUVs communicates with each other.

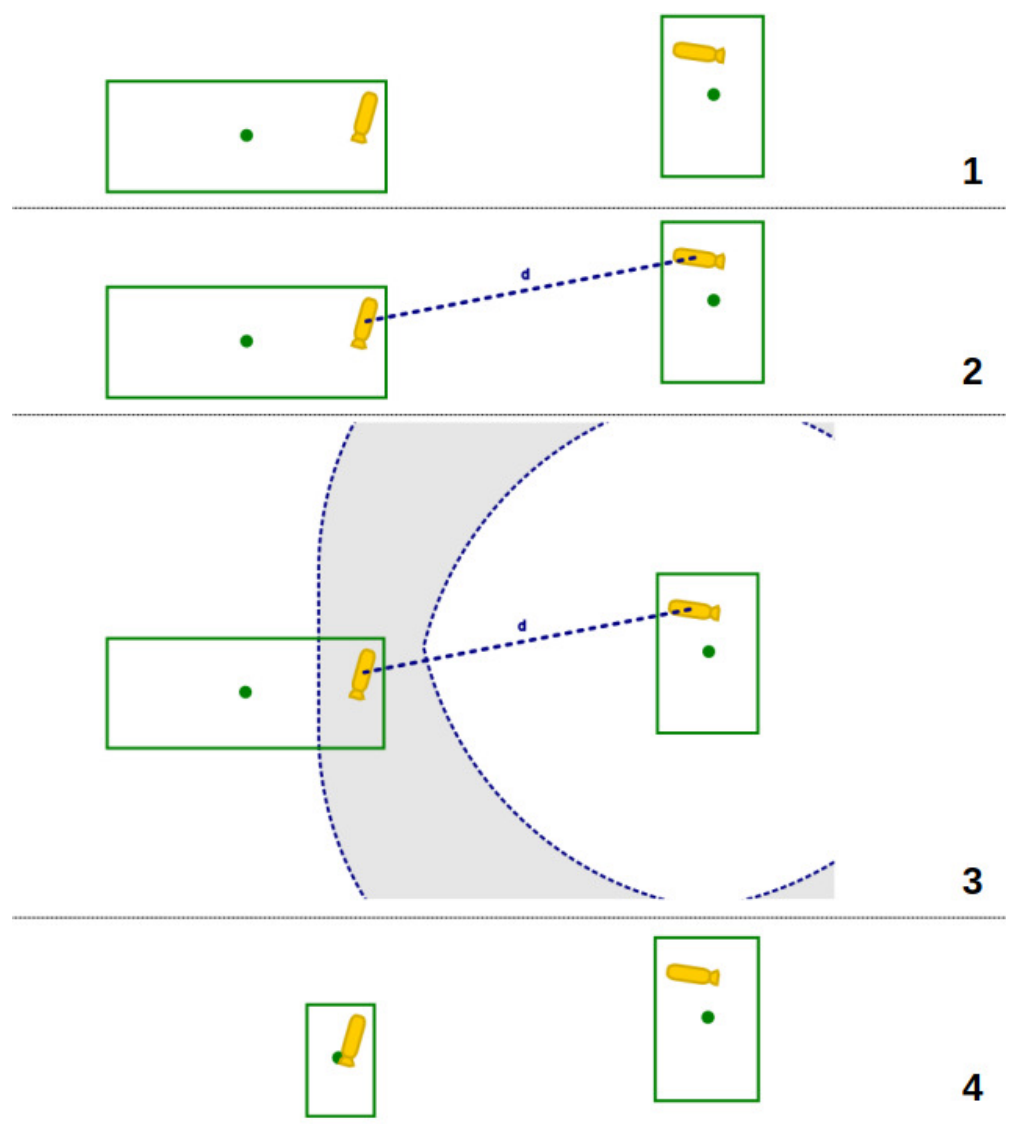

Figure 3. Localization accuracy procedure.
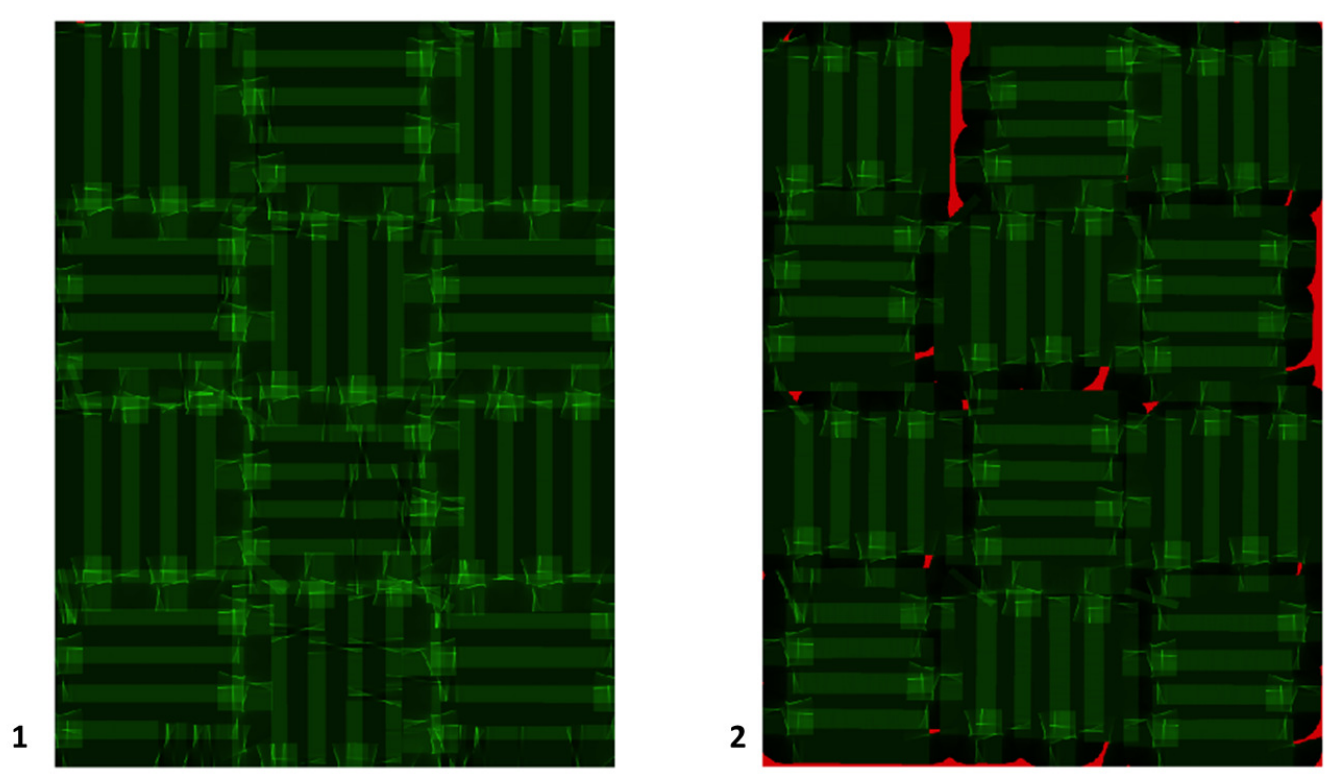

Figure 4. Area covered by a swarm of AUVs: left picture (number 1) is the coverage with communication, and the right picture (number 2) without communication. Red color represents no coverage, and the green intensity indicates the amount of scans on the area. 
Another benefit of having more precise positions, is that the collected data has a more accurate coordinates. As a consequence, the sweep of the surveyed area is less prone to samples gap.

Notice that all these advantages require a constant information exchange among the network nodes. The periodicity of the data exchange among AUVs depends on the mission, AUVs speed, amount of information, and distance among them. As an example, after theoretical studies which considered the projector bandwidth as $4 \mathrm{kHz}$, an useful modem bit rate of $2 \mathrm{kbps}$, and a maximum distance among the AUVs of $1 \mathrm{~km}$, the frame duration and the exchange periodicity were set to $2 \mathrm{~s}$ and $30 \mathrm{~s}$, respectively.

\section{Channel Access}

The communication among the AUVs is essential to accomplish any underwater task. During a mission, these vehicles need to exchange information for coordinating the navigation. Each AUV needs to compute its own position and velocity, and broadcast them to the other vehicles. So, the manner that these information will be exchanged among them, i.e., the channel access method, should be previously defined.

Many multiple access methods exist in the literature, such as time-division multiple access (TDMA), frequency-division multiple access (FDMA), code-division multiple access (CDMA), and Aloha. The Aloha protocol is efficient for the case the message length is proportionally smaller than the transmission periodicity. Although, this condition might not be satisfied in our case, specially when the number of AUVs increases.

In the CDMA method, each user is assigned with a spreading sequence to be used before transmitting the data. As the user can send information during all the time using the whole bandwidth, this system is highly susceptible to near-far effect. Despite that, it needs a power control mechanism at the transmitter side, which requires a feedback link. However, this is not desirable due to this additional resource consumption.

In the FDMA method, the frequency resources are shared among users. As the projector bandwith is small (only a few $\mathrm{kHz}$ ), each user will be assigned an even smaller bandwidth. This causes a reduction of the bit rate and subsequently an increase on the communication duration.

The TDMA method has an easy implementation and consists of sharing the time among users. One drawback of TDMA is that each AUV might have a distinct internal clock shift. Another inconvenience of utilizing TDMA for underwater communications is that the acoustic wave has a significant propagation delay. For coping with this channel feature, one should insert a guard interval, which reduces the information throughput. Nevertheless, TDMA was selected due to its robustness and resilience to near-far effects.

In the TDMA approach adopted in this project, each AUV can transmit information during a designated time slot, as shown in Figure 5. The communication ordering is previously defined, and each user needs to wait its turn for communicating. In this network, there is no central node, and each user has the knowledge of the total number of nodes.

All time slots have the same duration and its time length is defined as

$$
T_{\text {slot }}=T_{\text {frame }}+T_{\text {guard }}
$$

where $T_{\text {frame }}$ is the frame duration, and $T_{\text {guard }}$ is a guard interval. The frame duration is a fixed parameter and it is set to $T_{\text {frame }}=2 \mathrm{~s}$ [19]. The latter parameter $\left(T_{\text {guard }}\right)$ has the role of guaranteeing no transmission collision in the network. Notice that $T_{\text {guard }}$ can have a variable value, which depends on the channel and on the network topology. In other words, it depends on the maximum distance between two AUVs and on the highest channel delay spread:

$$
T_{\text {guard }}>\frac{d_{\max }}{c}+\tau_{\max }
$$


where $\tau_{\max }$ is the highest delay spread.
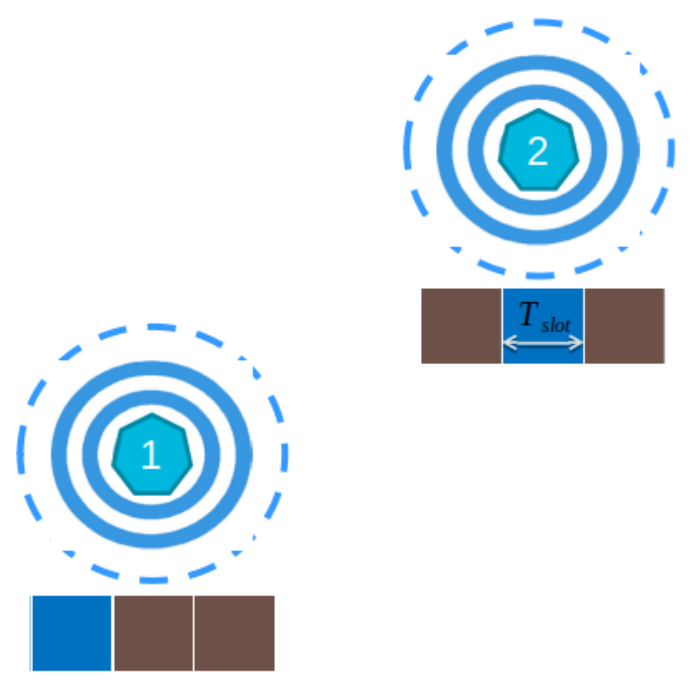

Figure 5. TDMA scheme.

\subsection{Importance of Adaptive Guard Interval}

We can define the percentage of the network usage as the time amount that a user is transmitting information over the total time slot that is allocated for that user:

$$
N_{\mathrm{u}}=\frac{T_{\text {frame }}}{T_{\text {frame }}+T_{\text {guard }}} .
$$

Ideally, it would be desirable that when an user stops transmitting, the next one could start immediately. As the acoustic wave has a low underwater propagation speed, the next node should wait that the most distant AUV receives all the signal.

For a better illustration, we consider that the guard interval is dominated by the term $d_{\max } / c$. We computed the effective network usage for distinct AUVs distance values. This result is depicted in Figure 6. In this figure, the x-axis is the real distance between the two most far away vehicles. The blue curve corresponds to the case that this information is perfectly known, leading to the usage of a $T_{\text {guard }}$ value which provides the maximum possible network usage rate. The left side of this curve, called the boundary, corresponds to the cases where the choice of $T_{\text {guard }}$ value results in no packet collision on the network. On the other hand, the usage of a $T_{\text {guard }}$ value which corresponds to an equivalent distance between transmitter and receiver lower than the real one, might result in packet collision.

In the same figure, we also plotted the network usage rate for the cases where the guard interval is set as a fixed parameter, independent of the network topology. As observed, this approach does not explore all the time resources (left side) or it prompts transmission collisions (right side). This leads to the necessity of a strategy such that the system can operate always under the blue curve, but the closest as possible to it. A possibility is to adapt this parameter according to the network topology. Knowing that each AUV periodically broadcasts its location and speed, we can take advantage of this data for adapting the guard interval. 


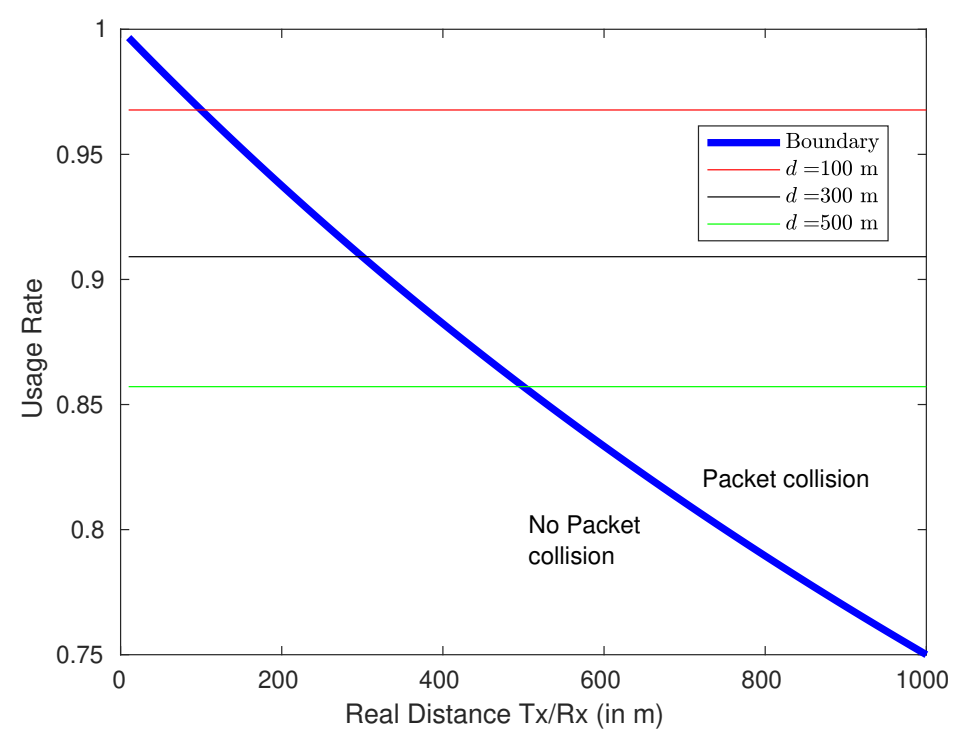

Figure 6. Network usage rate for $T_{\text {frame }}=2 \mathrm{~s}$.

\subsection{Frame Structure}

The frame structure employed for this swarm of AUVs is depicted in Figure 7. The information contained at each field is explained hereafter.

- Synchro: This field contains the synchronization signal, that is the first OFDM symbol. This initial signal is useful as well for the detection.

- @Dest: This is the identification of the message recipient (destinatary). Notice that each AUV is assigned with a distinct identification number. There is also an identification number for the case the message is designated for all users.

- @Srce: This field carries the source identification, i.e., the AUV that is currently transmitting information.

- Control: It is a control field, which can be used for many purposes. As an example, it might contain the congestion status of the local queue [19].

- Frame Number: This field contains the frame number.

- Hour: It contains the transmission time: hour, minutes, seconds, and milliseconds.

- Information Data: This field carriers the information data. For example, it might contain the information about the AUV position.

- $\quad$ FCS: This is a space reserved for a sequence that is added for detecting possible frame error transmission.

\begin{tabular}{|l|l|l|l|l|l|l|l|}
\hline Synchro & @dest & @srce & control & $\begin{array}{c}\text { Frame } \\
\text { Number }\end{array}$ & Time & Information Data & FCS \\
\hline
\end{tabular}

Figure 7. Frame structure.

\section{Physical Layer}

In digital communications, single carrier (SC) and OFDM waveforms are used to transmit information at a high data rate, and they have similar theoretical spectrum efficiency [20]. Both waveforms are impacted by the channel selectivity on time and frequency domains. For dealing with these channel impairments, specific signal processing techniques are required at the receiver side.

The underwater acoustic channel is highly time-variant, and its tracking can be a difficult task [21]. With this knowledge, it would be desirable that the selected waveform could cope with this inherent channel feature. The usage of a differential OFDM system does not require any channel equalization, decreasing the computational complexity at the receiver side [21]. Therefore, differential OFDM was selected for this project due to its 
robustness. Another motivation for this choice is the possibility of changing its parameters according to the application, and to the environmental conditions.

So, in this section, we present the physical layer aspect regarding the AUV's communication. We first describe the OFDM modem embedded in each AUV [22-24]. We then present a method that takes advantage of the knowledge of the network geometry to automatically adapt some of the OFDM parameters.

\subsection{Modem: Transmitter Side}

A block diagram of the transmitter is shown in Figure 8. The first procedure is the channel encoder. The information bits $\boldsymbol{b}_{i} \in\{0,1\}^{N}$ belonging to the each data block $i \in \mathbb{N}$ are coded:

$$
\overline{\boldsymbol{b}}_{i}=\mathcal{C}\left(\boldsymbol{b}_{i}, \rho\right),
$$

where $\mathcal{C}\left(\boldsymbol{b}_{i}, \rho\right)$ denotes the coding operation performed on $\boldsymbol{b}_{i}$, with code rate $\rho$. The result, $\overline{\boldsymbol{b}}_{i}$, is a vector containing $N / \rho$ coded bits (we consider that $N$ is an integer multiply of $\rho$ ). The benefit of utilizing a channel encoder is that this mechanism enables the receiver to detect and correct errors that might occur during the transmission. There are two distinct types of encoding available in the modem: the convolutional code, that is followed by an interleaver, and the Low-Density Parity-Check (LDPC) code.

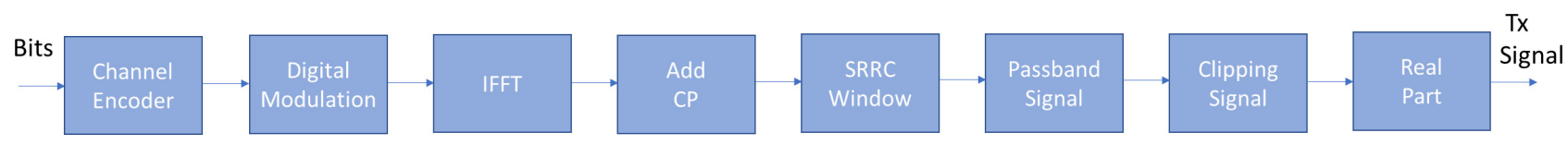

Figure 8. OFDM transmitter.

In the next stage, the coded bits $\bar{b}_{i}$ are mapped into symbols $s_{i}$ from a differential digital modulation:

$$
\boldsymbol{s}_{i}=\mathcal{M}\left(\overline{\boldsymbol{b}}_{i}, \mu\right)
$$

where $\mathcal{M}$ represents the differential digital modulation procedure, that maps $\mu$ bits into one complex modulated symbol. In this case, we employ either D-BPSK or D-QPSK modulation. The employment of differential modulation schemes enables the usage of a receiver with a lower complexity. For this modulation type, no channel equalization is required [21]. As a consequence, no pilot symbols are needed for channel equalization, reducing the overall system overhead [24].

These modulated symbols are mapped into the subcarriers through the multiplication by an IDFT matrix.

$$
\bar{s}_{i}=\boldsymbol{F}^{-1} \boldsymbol{s}_{i}
$$

where $\boldsymbol{F}^{-1} \in \mathbb{C}^{N_{\text {sub }} \times N_{\text {sub }} \text { is the IDFT matrix with }[\boldsymbol{F}]_{k, l}=\frac{1}{\sqrt{N_{\text {sub }}}} \mathrm{e}^{-\mathrm{j}} \frac{2 \pi}{N_{\text {sub }}} k l}$. We call $\overline{\boldsymbol{s}}_{i}$ as the $i$-th OFDM symbol.

Subsequently, a cyclic prefix (CP) with size $K$ is added to this signal, i.e., the last $K$ symbols are copied and added at the beginning of the OFDM symbol. The usage of a CP length higher or equal than the channel delay spread warrants that the receiver is able to remove the interblock interference (IBI), that the signal suffers while passing through the channel.

Following the proposal of [22,25], each OFDM block (that is the OFDM symbol along with its $\mathrm{CP}$ ), is multiplied by a squared root raised cosine (SRRC) pulse. In this windowing operation, which is performed over the whole OFDM block, the roll-off period is the same as the $\mathrm{CP}$ duration. As a consequence, some energy is saved [25]. In this pulse shaping design, the SRRC pulse is utilized both at transmitter and receiver side. 
This signal is modulated by the carrier generating the passband signal. Here, we denote as $f_{\mathrm{c}}$ the carrier frequency. For coping with peak-to-average power ratio (PAPR) issues, this signal is clipped. The real part of it is taken, and the signal is normalized before being transmitted.

\subsection{Modem: Receiver Side}

At the receiver side, the modem works under 3 distinct modes: the standby, locking and decoding mode [22]. These operation modes are depicted in Figure 9. The objective of having these three distinct modes is for power saving issues, without loosing any useful received information.

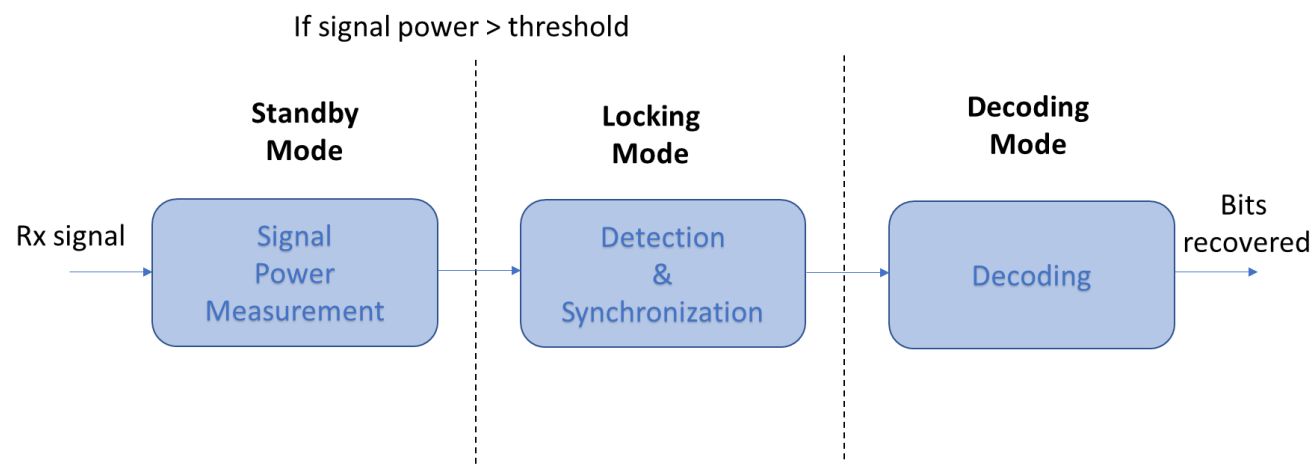

Figure 9. Receiver modes.

At the standby mode, the modem is continuously measuring the received signal power, and in case it detects an increase in the signal power, the locking mode is activated. In the locking mode, the signal of interest is detected and synchronized. The signal detection utilizes a maximum likelihood (ML) approach [24]. In this approach, a bank of matched filters is used for a joint estimation of the signal delay and of the Doppler factor. The first OFDM symbol is considered as a pilot, so that the filter bank is composed of distorted versions of this symbol. The selected branch is the one that provides the maximum value. The signal delay and the Doppler factor associated to this branch are chosen. The delay is used for synchronizing the received signal, and the Doppler factor is employed at a later processing stage.

After the signal be synchronized, the modem goes to the decoding mode, which is responsible for recovering the transmitted signal. Figure 10 shows the block diagram of the receiver when operating at this mode.

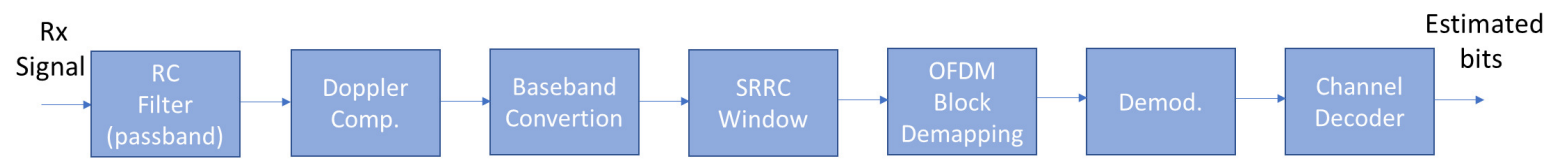

Figure 10. Modem: receiver operating at decoding mode.

In order to keep only the desired signal, which has a bandwidth of $B=M \Delta f$ and is centered in $f_{c}$, the received signal passes through a passband filter. This is a raised cosine filter, and in Figure 10 it is denoted as RC filter.

Notice that the received signal samples, do not correspond to the originally equally spaced transmitted samples [26]. This phenomenon is due to the Doppler effect, which introduces a time-warping effect on the signal, i.e., the signal suffers a compression and/or dilatation in the time domain. For compensating the Doppler effect, the following procedure is performed. Knowing the time instant that each signal sample was received, and the initial Doppler factor (that was obtained at the locking mode), we can recover the 
original transmission time instant. With this information, the received signal is resampled for finding the original equally spaced signal.

At the next stage, the carrier frequency is removed, and the signal is shifted to baseband. This signal is multiplied by a squared-root raised cosine window [22]. This apodization operation is part of the pulse shaping design [25], which enables a reduction on the power consumption.

Then this signal passes through a serial to parallel converter. At the S/P converter output, we will have an OFDM block, which contains $\left(N_{\text {sub }}+K\right)$ symbols. So, this OFDM block is demapped, leading to approximations of the differential complex modulated symbols. These symbols are demodulated, giving rise to bits. At the next stage, the set of bits is decoded, leading to the estimated transmitted bits.

\subsection{Adaptive Parameters}

The modem previously described is embedded in each AUV. When the AUV is operating in a swarm mode, the system dynamics are constantly changing. So, it would be beneficial if each vehicle could automatically adapt some physical layer parameters according to the channel.

When a feedback link is available, the physical layer adaptation is getting some kind of channel state information from the receiver $[6-9,12,13]$. Such an approach can be efficient but is costly in terms of overhead and energy. Apart from that, a feedback link might not be feasible for some underwater applications, such as long range communications [14].

In our context, no feedback is provided. However, we can take advantage of the information of the AUVs location and speed that is periodically broadcasted. Based on this knowledge, we propose to run, at the transmitter side, an on-board channel simulator. The outputs of this simulated channel are then used to predict some metrics on the modem performance. OFDM parameters that can be adjusted include the number of subcarriers $\left(N_{\text {sub }}\right)$ and the cyclix prefix length $\left(T_{\mathrm{CP}}\right)$. In order to correctly adapt these parameters, it is important to first understand how they impact the system performance.

Considering a fixed bandwidth, the usage of a large number of subcarriers will decrease the subcarrier spacing. If this spacing is small enough, the information recovery at the receiver side can be compromised. For example, the Doppler effect might cause intercarrier interference (ICI), and as a consequence the subcarriers orthogonality is lost, hindering the information recovery. On the other hand if we choose a low number of subcarriers, the resulting throughput is lower than the one that could be achieved (due to the CP that is added in each OFDM symbol). So, a proper choice of $N_{\text {sub }}$ is of paramount importance for an efficient resource usage.

Knowing that the underwater acoustic communication is impaired by the multipath channel with a large delay spread, one must consider these effects when designing a communication system. A possible solution to tackle this issue is to add a cyclic prefix (CP) at the signal before transmission. The main function of $\mathrm{CP}$ is to remove the interblock interference (IBI) at the receiver side. Although, if the CP length is lower than the channel delay spread, the IBI is not eliminated. Still, if the $\mathrm{CP}$ is much longer, we are wasting power and transmission time.

A possible way of choosing the number of subcarriers and the $\mathrm{CP}$ length would be through the minimization of the IBI and the ICI [27]. As the CP length has a direct impact on the transmission rate, we decided to constraint the system overhead coming from this parameter. So, this optimization problem can be mathematically written as

$$
\begin{aligned}
\min _{N_{\mathrm{sub},}, T_{\mathrm{CP}}} & \left(P_{\mathrm{ICI}}+P_{\mathrm{IBI}}\right) \\
\text { s.t. } & \rho \leq \rho_{\max }
\end{aligned}
$$


where $P_{\mathrm{ICI}}, P_{\mathrm{IBI}}$ are the signal power containing ICI and IBI, respectively. The value $\rho_{\max }$ is chosen by the user, and it represents the maximum overhead due to CP. The overhead $\rho$ is defined as

$$
\rho=\frac{T_{\mathrm{CP}}}{T_{\mathrm{CP}}+T_{\mathrm{sym}}},
$$

where $T_{\text {sym }}$ is the useful symbol duration.

The low speed propagation of the acoustic waves increases the round-trip time [26], which may reflect in variations of the channel impulse response. So, it might not be feasible to use channel state information (CSI) for computing $P_{\mathrm{ICI}}$ and $P_{\mathrm{IBI}}$.

A possibility is to predict the interference power due to ICI using [28]. In addition, since no feedback link is available, the interference can only be predicted but cannot be estimated. In reference [28], the bound for $P_{\mathrm{ICI}}$ is derived for an OFDM system considering a time-variant channel, and its resulting expression is given by

$$
P_{\mathrm{ICI}}=1-\int_{-f_{d}}^{f_{d}} P(f) \operatorname{sinc}^{2}\left(f T_{\mathrm{sym}}\right) \mathrm{d} f,
$$

with $f_{d}$ being the value of the maximum Doppler frequency, and $P(f)$ the Doppler spectrum. The variable $f_{d}$ is defined as

$$
f_{d}=\frac{v}{c} \cdot f_{\mathrm{c}}
$$

where $v$ is the relative velocity between transmitter and receiver, and $c$ is the sound speed propagation. Since the actual Doppler spectrum $P(f)$ is unknown, it is predicted. This prediction is made using the maximum entropy principle [29]. The main motivation for using this approach is to avoid adding random and unknown information to the system. These models are specially useful in underwater applications [29-31]. For this work, we assume that the Doppler spectrum $P(f)$ has a known bounded normalized support. In other words, we consider as known the maximum Doppler effect. The computation of $P(f)$ follows the guidelines provided at [29], in which is employed a numerical approach.

Knowing that we utilize a full redundancy receiver, we can compute the interference power due to IBI as follows:

$$
P_{\mathrm{IBI}}=\int_{T_{\mathrm{CP}}}^{+\infty} P_{d p}(\tau) \mathrm{d} \tau,
$$

with $P_{d p}(\tau)$ being the channel power-delay profile. For obtaining a prediction of the power delay-profile, we utilize a wideband ray tracer for shallow water [32]. In order to also consider the frequency dependent attenuation phenomenon, Thorp's formula [33] is added to this procedure.

For solving the optimization problem of Equation (7), we compute the interference power for all predefined pairs of parameters $\left(N_{\mathrm{sub}}, T_{\mathrm{CP}}\right)$. The pair which leads to the minimum interference, and that obeys the overhead constraint is selected.

For summarizing, Table 1 shows all the input parameters of the optimization problem. Notice that the optimization only requires knowledge about the channel geometry. As output, the optimization algorithm provides the number of subcarriers and the $\mathrm{CP}$ length $\left(N_{\text {sub }}, T_{\mathrm{CP}}\right)$, as shown in Table 2. 
Table 1. Optimization input parameters.

\begin{tabular}{cc}
\hline & Input Parameters \\
\hline$f_{\mathrm{c}}$ & Carrier frequency (in Hz) \\
$B$ & System bandwidth (in Hz) \\
$c$ & Sound speed (in $\mathrm{m} / \mathrm{s})$ \\
$v$ & Relative velocity Tx/Rx (in m/s) \\
$p_{\mathrm{w}}$ & Water depth (in $\mathrm{m})$ \\
$p_{\mathrm{Tx}}$ & Transmitter depth (in m) \\
$p_{\mathrm{Rx}}$ & Receiver depth (in m) \\
$d_{\mathrm{Tx}, \mathrm{Rx}}$ & Distance Tx/Rx (in m) \\
$\rho_{\max }$ & Maximum overhead (constraint) \\
\hline
\end{tabular}

Table 2. Optimization output parameters.

\begin{tabular}{cc}
\hline & Output Parameters \\
\hline$N_{\text {sub }}$ & number of subcarriers \\
$T_{\mathrm{CP}}$ & CP duration (in ms) \\
\hline
\end{tabular}

\subsection{Optimization Evaluation}

For assessing the performance of this optimization method, we compared its results with a genie-aided modem. This latter approach selects the parameters pair $\left(N_{\mathrm{sub}}, T_{\mathrm{CP}}\right)$ which leads to the minimum measured bit-error rate (BER). For this case, we utilized the previously described modem. All the tested cases considered a D-BPSK constellation and a sampling rate $f_{s}=192 \mathrm{kHz}$. We utilize the Watermark simulator [34,35] for replaying the underwater channel. This simulator provides real measurements of the channels, which have a time-varying impulse response. Performance is first evaluated without channel coding and without noise in the channel. Results with coding and noise are then discussed in Section 5.5. The Matlab program was utilized for performing all these simulations.

\subsubsection{BCH1 Channel}

The first selected scenario concerns the BCH1 (Brest Commercial Harbor) channel $[34,35]$. This experiment was performed in France, and the transmitter was placed $800 \mathrm{~m}$ away from the receiver. The channel geometry data regarding this experiment is shown in Table 3.

Table 3. BCH1 channel conditions used for the optimization.

\begin{tabular}{cc}
\hline & Input Parameters \\
\hline$f_{\mathrm{c}}$ & $35 \mathrm{kHz}$ \\
$B$ & $4 \mathrm{kHz}$ \\
$c$ & $1500 \mathrm{~m} / \mathrm{s}$ \\
$v$ & $0.1 \mathrm{~m} / \mathrm{s}$ \\
$p_{\mathrm{w}}$ & $20 \mathrm{~m}$ \\
$p_{\mathrm{Tx}}$ & $2 \mathrm{~m}$ \\
$p_{\mathrm{Rx}}$ & $5 \mathrm{~m}$ \\
$d_{\mathrm{Tx}, \mathrm{Rx}}$ & $800 \mathrm{~m}$ \\
\hline
\end{tabular}

For running the optimization algorithm, we restricted the set of possible subcarriers value and the $C P$ lengths. In this case, we consider $N_{\text {sub }}=\{128,256,512\}$ subcarriers, and $T_{\mathrm{CP}}=\{1,5,8,10,12,15,18\} \mathrm{ms}$. Notice that the OFDM symbol duration (without $\mathrm{CP}$ ) varies according to the number of subcarriers selected. So, using these information, the optimization algorithm is executed. The obtained results are depicted in Table 4. This table contains the optimized parameters for distinct overhead constraints. 
Table 4. Optimized parameters for BCH1 channel.

\begin{tabular}{cccccc}
\hline & \multicolumn{5}{c}{ Maximum Overhead $\left(\rho_{\max }\right)$} \\
\cline { 2 - 6 } & $\mathbf{0 . 1}$ & $\mathbf{0 . 1 5}$ & $\mathbf{0 . 2}$ & $\mathbf{0 . 2 5}$ & $\mathbf{0 . 3}$ \\
\hline$T_{\mathrm{CP}}(\mathrm{ms})$ & 5 & 10 & 8 & 10 & 12 \\
$N_{\text {sub }}$ & 256 & 256 & 128 & 128 & 128 \\
$T_{\text {sym }}$ & 64 & 64 & 32 & 32 & 32 \\
\hline
\end{tabular}

In order to evaluate these optimized results, we ran other simulations with a genieaided modem. For this comparative approach, it was transmitted 27 frames, whereas each frame had a $1 \mathrm{~s}$ time length. This procedure was repeated 50 times and the results are in Figure 11. This figure shows the bit-error rate (BER) obtained for all possible system configurations. An explanation for the system behavior shown in Figure 11 is as follows. Considering the same bandwidth, the usage of a larger number of subcarriers translates to a lower subcarrier spacing. When the subcarrier spacing is small, the system is more susceptible to ICI, resulting in a increase of the BER. On the other hand, the augmentation of $\mathrm{CP}$ length improves the system performance. The decrement on the BER value can be explained by a reduction on the IBI at the receiver side. Although, this behavior is not so explicit for $N_{\text {sub }}=512$. In this case, the ICI dominates, masking the influence of the $\mathrm{CP}$ length.

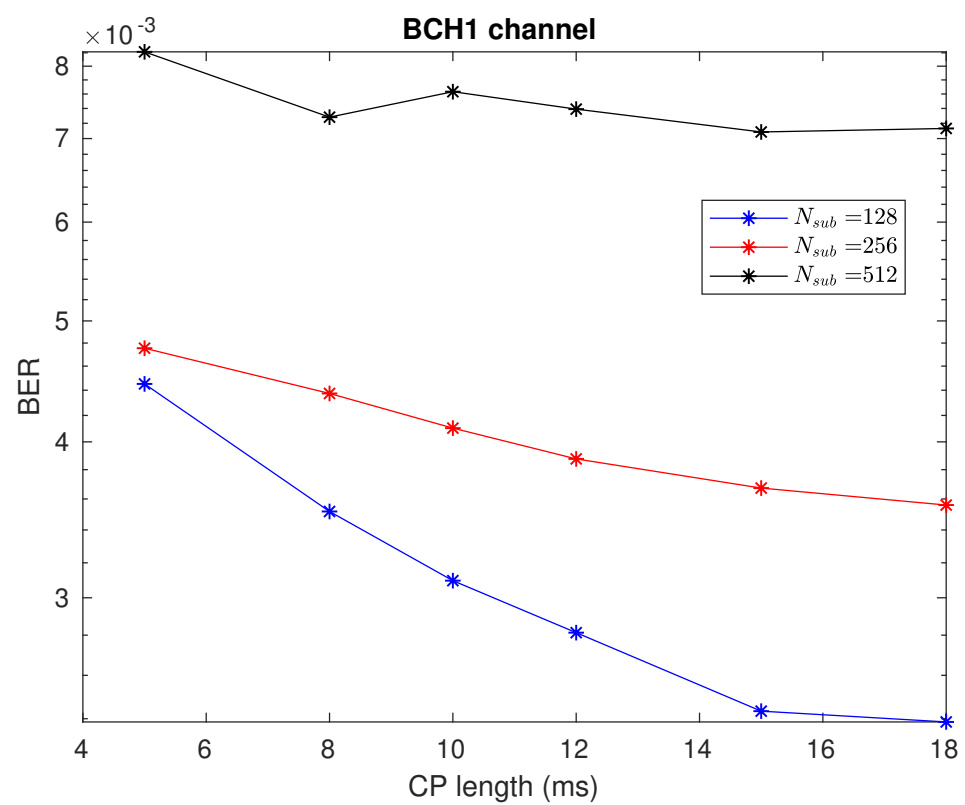

Figure 11. Results for $\mathrm{BCH} 1$ channel considering the genie-aided approach.

In this genie-aided approach, the parameters pair to be selected should also obey the overhead constraint. As each point from Figure 11 has a distinct overhead value associated, we should check the eligibility of each point. Figure 12 shows the overhead inherent to each point. In this figure it is possible to observe that a higher number of subcarriers is associated with a lower overhead. This behavior is due to the fact that the OFDM symbol duration (without CP) depends on the number of subcarriers. The larger the number of subcarriers, the longer the OFDM symbol duration. 


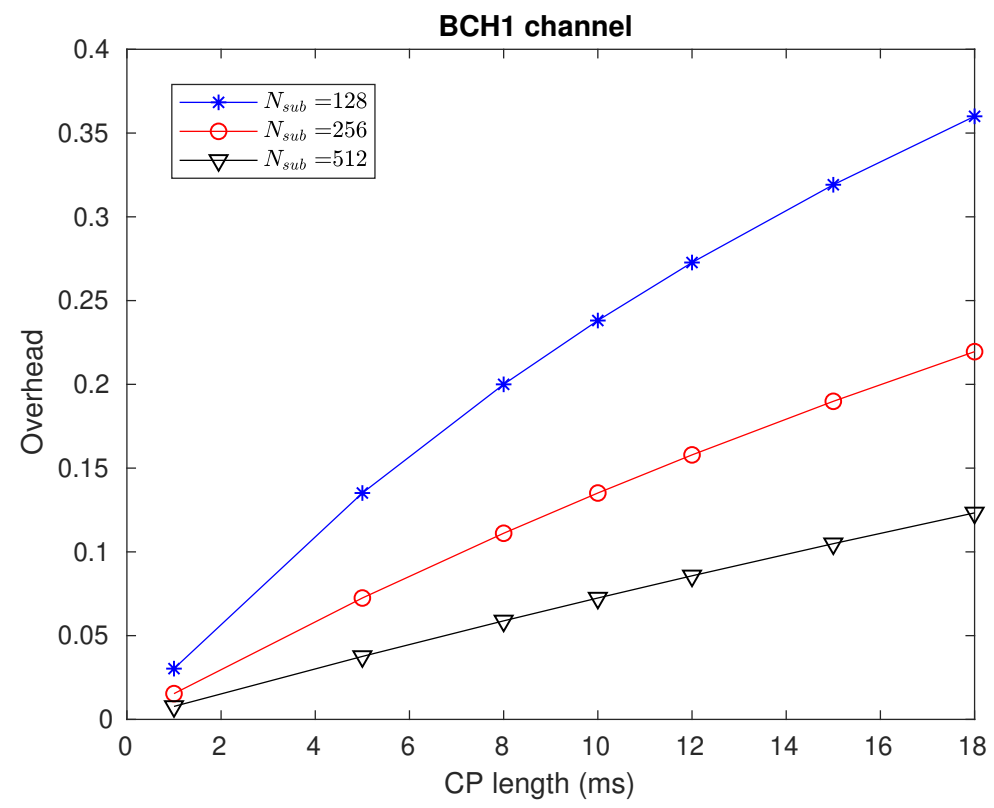

Figure 12. Corresponding overhead for each parameters pair for $\mathrm{BCH} 1$ channel.

So, the selection of a benchmark point depends on the desirable overhead value. As an example, for an overhead of $\rho_{\max }=0.1$, the following points are eligible (see Figure 12): $\left(N_{\text {sub }}=128, T_{\mathrm{CP}}=1\right),\left(N_{\text {sub }}=256, T_{\mathrm{CP}}=1\right),\left(N_{\text {sub }}=256, T_{\mathrm{CP}}=5\right),\left(N_{\text {sub }}=512, T_{\mathrm{CP}}=1\right)$, $\left(N_{\text {sub }}=512, T_{\mathrm{CP}}=5\right),\left(N_{\mathrm{sub}}=512, T_{\mathrm{CP}}=8\right),\left(N_{\mathrm{sub}}=512, T_{\mathrm{CP}}=10\right),\left(N_{\mathrm{sub}}=512\right.$, $\left.T_{\mathrm{CP}}=12\right)$. Considering only these points, we choose the parameters pair that has the lowest BER in Figure 11. For this case, the selected point is $(256,10)$.

The above-mentioned procedure was performed for all overhead values considered in Table 4, and these results are shown in Table 5. As observed, all the parameters pair obtained by the optimization algorithm are the same as the benchmark points.

Table 5. Parameters chosen by each method for BCH1 channel.

\begin{tabular}{cccccc}
\hline & \multicolumn{5}{c}{ Max. Overhead $\left(\rho_{\text {max }}\right)$} \\
\cline { 2 - 6 } & $\mathbf{0 . 1}$ & $\mathbf{0 . 1 5}$ & $\mathbf{0 . 2}$ & $\mathbf{0 . 2 5}$ & $\mathbf{0 . 3}$ \\
\hline Proposed method & $(256,5)$ & $(256,10)$ & $(128,8)$ & $(128,10)$ & $(128,12)$ \\
Genie-aided method & $(256,5)$ & $(256,10)$ & $(128,8)$ & $(128,10)$ & $(128,12)$ \\
\hline Equal results & yes & yes & yes & yes & yes \\
\hline
\end{tabular}

\subsubsection{KAU1 Channel}

Another considered scenario is the KAU1 channel (Kauai Acomms MURI 2011) [34,35]. This channel was recorded in Kauai, HI, USA. All the information relative to this experiment, and that are needed for the optimization procedure, are shown in Table 6.

Table 6. KAU1 channel conditions used for the optimization.

\begin{tabular}{cc}
\hline & Input Parameters \\
\hline$f_{\mathrm{c}}$ & $6 \mathrm{kHz}$ \\
$B$ & $3 \mathrm{kHz}$ \\
$c$ & $1500 \mathrm{~m} / \mathrm{s}$ \\
$v$ & $1.14 \mathrm{~m} / \mathrm{s}$ \\
$p_{\mathrm{w}}$ & $106 \mathrm{~m}$ \\
$p_{\mathrm{Tx}}$ & $45 \mathrm{~m}$ \\
$p_{\mathrm{Rx}}$ & $97 \mathrm{~m}$ \\
$d_{\mathrm{Tx}, \mathrm{Rx}}$ & $1080 \mathrm{~m}$ \\
\hline
\end{tabular}


Having this information, the optimization algorithm is executed. For this case, the following values were considered: $N_{\text {sub }}=\{128,256,512\}$ subcarriers, and $T_{\mathrm{CP}}=$ $\{5,10,18,25,30,35,41\} \mathrm{ms}$. The optimized results are presented in Table 7.

Table 7. Optimization parameters considering the KAU1 channel.

\begin{tabular}{cccccc}
\hline & \multicolumn{5}{c}{ Maximum Overhead $\left(\rho_{\max }\right)$} \\
\cline { 2 - 6 } & $\mathbf{0 . 1}$ & $\mathbf{0 . 1 5}$ & $\mathbf{0 . 2}$ & $\mathbf{0 . 2 5}$ & $\mathbf{0 . 3}$ \\
\hline$T_{\mathrm{CP}}(\mathrm{ms})$ & 18 & 10 & 10 & 25 & 35 \\
$N_{\text {sub }}$ & 512 & 256 & 128 & 256 & 256 \\
$T_{\text {sym }}(\mathrm{ms})$ & 170.6 & 85.3 & 42.6 & 85.3 & 85.3 \\
\hline
\end{tabular}

For evaluating these optimized results, simulations using the genie-aided approach were performed. We transmitted 14 frames with a $1 \mathrm{~s}$ duration. Each simulation was repeated 50 times, and the results are shown in Figure 13. Figure 14 contains the overhead value associated to each point of the previous figure. So, the benchmark parameters are selected for each overhead value.

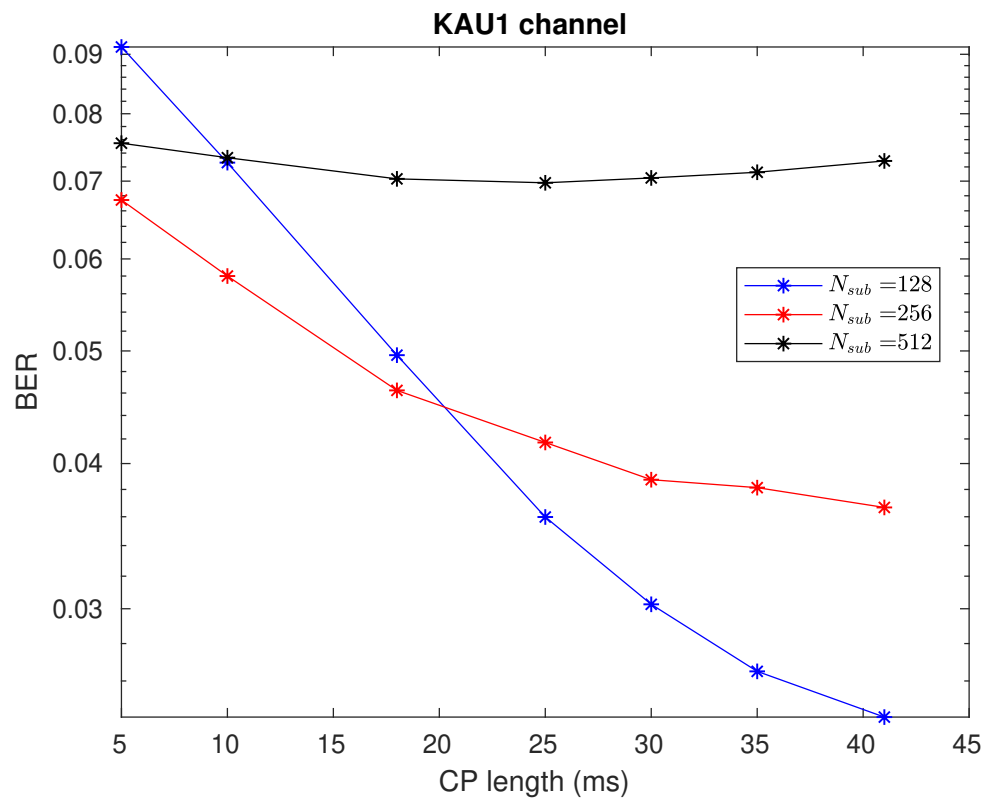

Figure 13. Results for KAU1 channel considering the genie-aided approach.

Table 8 shows results for both optimized and benchmark approaches. The majority of the parameters obtained with our method matches with the genie-aided results.

Table 8. Parameters chosen by each method for KAU1 channel.

\begin{tabular}{cccccc}
\hline & \multicolumn{5}{c}{ Max. Overhead $\left(\rho_{\text {max }}\right)$} \\
\cline { 2 - 6 } & $\mathbf{0 . 1}$ & $\mathbf{0 . 1 5}$ & $\mathbf{0 . 2}$ & $\mathbf{0 . 2 5}$ & $\mathbf{0 . 3}$ \\
\hline Proposed method & $(512,18)$ & $(256,10)$ & $(128,10)$ & $(256,25)$ & $(256,35)$ \\
Genie-aided method & $(256,5)$ & $(256,10)$ & $(256,10)$ & $(256,25)$ & $(256,35)$ \\
\hline Equal results & no & yes & no & yes & yes \\
\hline
\end{tabular}




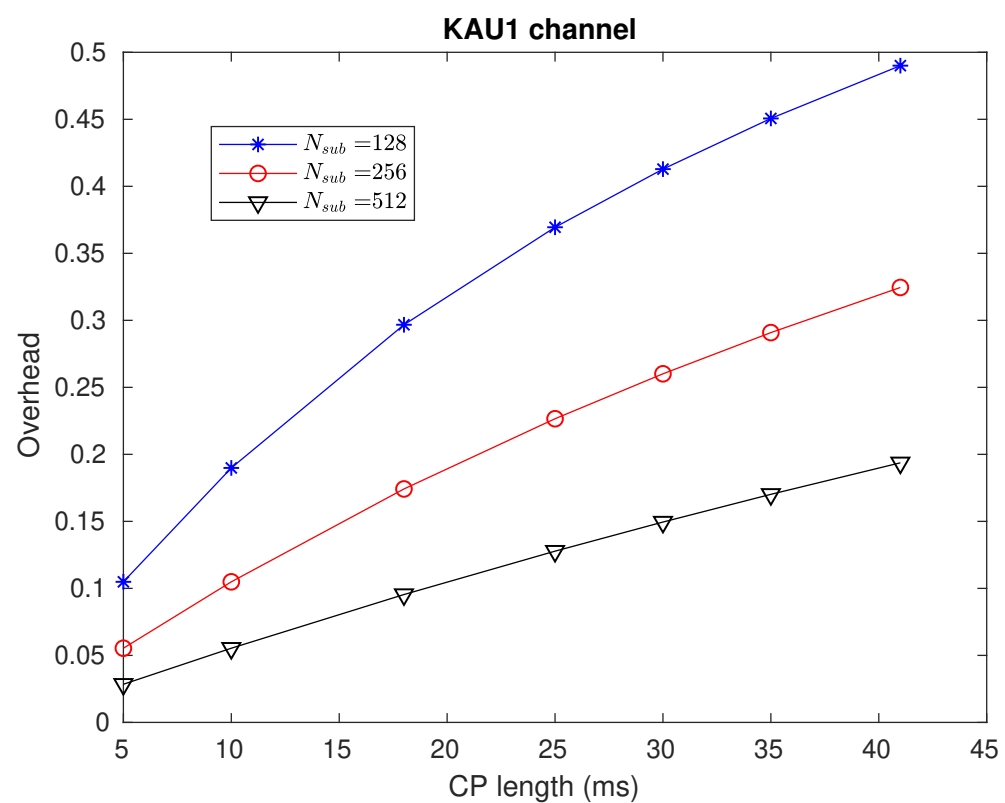

Figure 14. Corresponding overhead for each parameters pair for KAU1 channel.

\subsection{Modem Performance with Adaptive Parameters}

In order to evaluate the system performance when the modem operates with the optimization procedure, we performed simulations in a noisy environment. In this case, the system utilized channel encoder. The Watermark simulator $[34,35]$ was utilized for emulating the channel. We added white Gaussian noise to the signal that passed through the channel. The signal-to-noise ratio (SNR) is measured at the receiver side, and it is defined as:

$$
S N R=10 \log _{10}\left(\frac{E_{\mathrm{b}}}{N_{0}}\right)
$$

where $E_{\mathrm{b}}$ is the bit energy, and $N_{0}$ is the noise power spectral density.

The modem was set with the following parameters for the simulations performed considering this framework. The channel encoder utilized is the convolutional, with a code rate of $1 / 2$, and constraint length 7 . The bits are modulated with a D-QPSK modulation, and the sampling rate is $f_{s}=192 \mathrm{kHz}$. In addition to that, each simulation is repeated 100 times. For each channel, and for a given overhead value, the optimization algorithm is executed for obtaining the number of subcarriers $\left(N_{\mathrm{sub}}\right)$ and the cyclic prefix length $\left(T_{\mathrm{CP}}\right)$.

We ran simulations regarding the environment of KAU1 channel. Figure 15 shows the BER as a function of the SNR. The curves considering the optimized points are indicated in the legend as $\rho_{\max }=0.15$ and $\rho_{\max }=0.3$. The other two curves were generated considering a parameters pair randomly selected, i.e., the unoptimized case. For $\rho_{\max }=0.15$, it is possible to observe the performance gain when utilizing the optimization algorithm. This gain increases with the augmentation of the SNR. The same behavior is observed for $\rho_{\max }=0.3$. In this case, the gain is even more significant: for SNR values higher than $12 \mathrm{~dB}$, the BER is improved in at least one order of magnitude. 


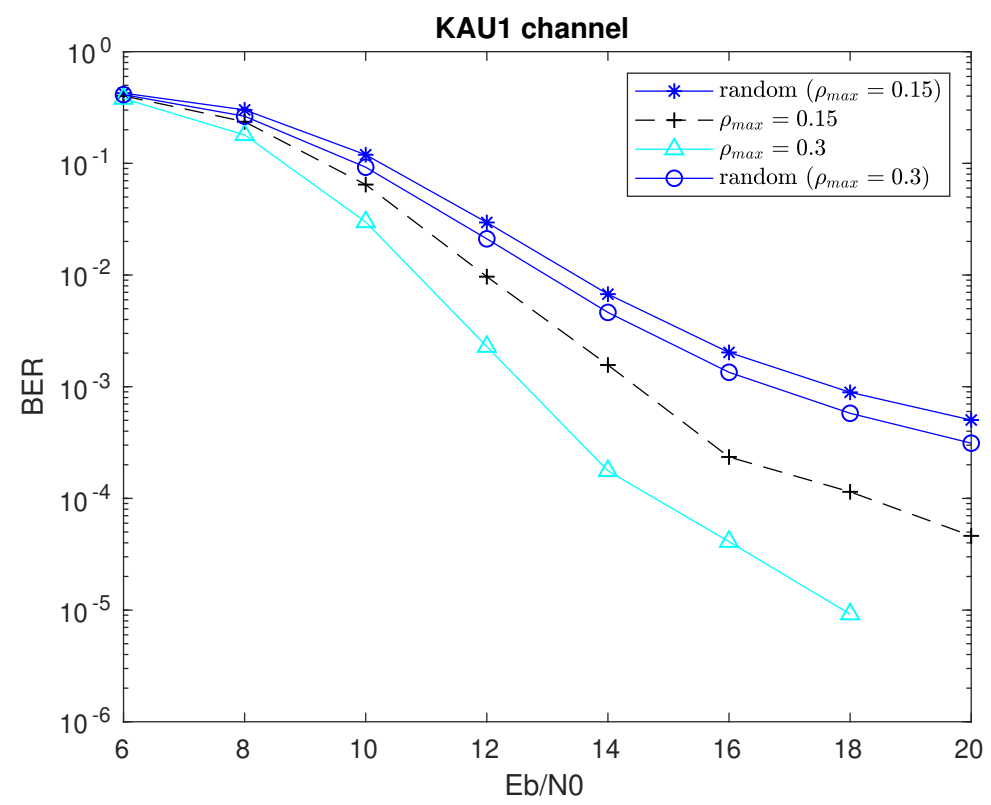

Figure 15. Modem operating at KAU1 channel, and for distinct maximum overhead values.

All these results show the benefits the optimization algorithm can bring to the system.

\section{Conclusions}

In this paper we addressed all the AUVs swarm communication process developed for COMET and NEMOSENS projects, with a focus on the proposal of a method for adapting some physical layer parameters. We discussed about the achievable benefits when these vehicles can exchange information among them. The usage of underwater communications improves navigation and localization in an AUV swarm. In addition to that, we showed our designed communication process for an underwater AUVs network. At first, we presented the selected channel access method, and we proposed the usage of an adaptive guard interval for improving the network usage rate. We also addressed all the aspects concerning the physical layer. We explained the modem employed at the transmitter and receiver side, along with its operation modes. Subsequently, we proposed an optimization method for adapting some physical layer parameters: the number of subcarriers $\left(N_{\mathrm{sub}}\right)$ and the cyclic prefix length $\left(T_{\mathrm{CP}}\right)$. This method utilizes only information about the transmission geometry and does not require any kind of feedback information. The idea behind this approach is to utilize the location and speed information that each AUV periodically broadcasts. We showed that this optimization is able to provide similar results as the ones obtained by a genie-aided approach. At the end, we presented results for the case the modem is operating with this optimization method. These results, utilizing the Watermark simulator, show a performance improvement in terms of bit-error rate. We intend to perform sea-experiments for evaluating the benefits of this new optimization feature.

Author Contributions: Conceptualization, F.-X.S., B.Z. and T.L.M.; Funding acquisition, R.B.; Project administration, C.L.B.; Supervision, C.L.; Validation, C.L., F.-X.S., B.Z., T.L.M. and R.B.; Writingoriginal draft, C.M.G.G.; Writing-review \& editing, C.L. and F.-X.S. All authors have read and agreed to the published version of the manuscript.

Funding: This research was funded by Region Bretagne and BPI France.

Conflicts of Interest: The authors declare no conflict of interest. 


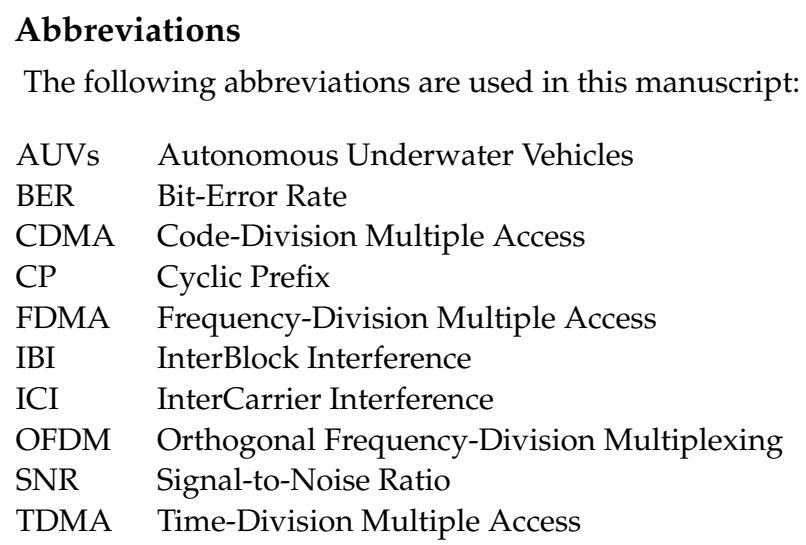

\section{References}

1. Petillo, S.; Schmidt, H.; Balasuriya, A. Constructing a Distributed AUV Network for Underwater Plume-Tracking Operations. Int. J. Distrib. Sens. Netw. 2012, 8, 191235. [CrossRef]

2. Burrowes, G.E.; Brown, J.; Khan, J.Y. Adaptive Space Time-Time Division Multiple Access (AST-TDMA) protocol for an underwater swarm of AUV's. In Proceedings of the 2013 MTS/IEEE OCEANS, Bergen, Norway, 10-14 June 2013; pp. 1-10.

3. Ferri, G.; Munafò, A.; Tesei, A.; Braca, P.; Meyer, F.; Pelekanakis, K.; Petroccia, R.; Alves, J.; Strode, C.; LePage, K. Cooperative robotic networks for underwater surveillance: An overview. IET Radar Sonar Navig. 2017, 11, 1740-1761. [CrossRef]

4. Şahin, E. Swarm Robotics: From Sources of Inspiration to Domains of Application; Springer: Berlin/Heidelberg, Germany, 2005; pp. 10-20.

5. Sabra, A.; Fung, W.-K. A Fuzzy Cooperative Localisation Framework for Underwater Robotic Swarms. Sensors 2020, 20 , 5496. [CrossRef]

6. Radosevic, A.; Ahmed, R.; Duman, T.M.; Proakis, J.G.; Stojanovic, M. Adaptive OFDM Modulation for Underwater Acoustic Communications: Design Considerations and Experimental Results. IEEE J. Ocean. Eng. 2014, 39, 357-370. [CrossRef]

7. Wan, L.; Zhou, H.; Xu, X.; Huang, Y.; Zhou, S.; Shi, Z.; Cui, J.H. Adaptive Modulation and Coding for Underwater Acoustic OFDM. IEEE J. Ocean. Eng. 2015, 40, 327-336. [CrossRef]

8. Huang, L.; Zhang, Q.; Zhang, L.; Shi, J.; Zhang, L. Efficiency Enhancement for Underwater Adaptive Modulation and Coding Systems: Via Sparse Principal Component Analysis. IEEE Commun. Lett. 2020, 24, 1808-1811. [CrossRef]

9. Fu, Q.; Song, A. Adaptive Modulation for Underwater Acoustic Communications Based on Reinforcement Learning. In Proceedings of the 2018 MTS/IEEE OCEANS, Charleston, SC, USA, 22-25 October 2018; pp. 1-8.

10. Su, W.; Lin, J.; Chen, K.; Xiao, L.; En, C. Reinforcement Learning-Based Adaptive Modulation and Coding for Efficient Underwater Communications. IEEE Access 2019, 7, 67539-67550. [CrossRef]

11. Alamgir, M.S.M.; Sultana, M.N.; Chang, K. Link Adaptation on an Underwater Communications Network Using Machine Learning Algorithms: Boosted Regression Tree Approach. IEEE Access 2020, 8, 73957-73971. [CrossRef]

12. Pottier, A.; Socheleau, F.; Laot, C. Quality-of-Service Satisfaction Games for Noncooperative Underwater Acoustic Communications. IEEE Access Top. Underw. Wirel. Commun. Netw. 2018, 6, 21467-21481. [CrossRef]

13. Pottier, A.; Socheleau, F.; Laot, C. Robust Noncooperative Spectrum Sharing Game in Underwater Acoustic Interference Channels. IEEE J. Ocean. Eng. 2017, 42, 1019-1034. [CrossRef]

14. Huang, J.; Diamant, R. Adaptive Modulation for Long-Range Underwater Acoustic Communication. IEEE Trans. Wirel. Commun. 2020, 19, 6844-6857. [CrossRef]

15. COMET Briefsheet. Available online: https://rtsys.eu/wp-content/uploads/2021/01/COMET_300_Briefsheet_003.pdf (accessed on 30 June 2021).

16. NemoSens Briefsheet. Available online: https://rtsys.eu/wp-content/uploads/2021/02/NemoSens_Briefsheet_EN_010.pdf (accessed on 30 June 2021).

17. González-García, J.; Gómez-Espinosa, A.; Cuan-Urquizo, E.; García-Valdovinos, L.G.; Salgado-Jiménez, T.; Cabello, J.A.E. Autonomous Underwater Vehicles: Localization, Navigation, and Communication for Collaborative Missions. Appl. Sci. 2020, 10, 1256. [CrossRef]

18. Drevelle, V.; Jaulin, L.; Zerr, B. Set membership approach for underwater exploration with swarms. In Proceedings of the International Conference on Underwater Remote Sensing, Brest, France, 8-11 October 2012.

19. Lmai, S.; Houcke, S.; Laot, C.; Bourré, A. Projet COMET: Développement du Schéma D'accès au Canal; Technical Report; Telecom Bretagne: Brest, France, 2013.

20. Li, Y.G.; Stüber, G. Orthogonal Frequency Division Multiplexing for Wireless Communications; Springer: Berlin/Heidelberg, Germany, 2006.

21. Aval, Y.; Stojanovic, M. Differential OFDM for acoustic communications. J. Acoust. Soc. Am. 2012, 131, 3238. [CrossRef]

22. Bourré, A.; Lmai, S.; Laot, C.; Houcke, S. A robust OFDM modem for underwater acoustic communications. In Proceedings of the 2013 MTS/IEEE OCEANS, Bergen, Norway, 10-14 June 2013; pp. 1-5.

23. Lmai, S.; Bourré, A.; Laot, C.; Houcke, S. An Efficient Blind Estimation of Carrier Frequency Offset in OFDM Systems. IEEE Trans. Veh. Technol. 2014, 63, 1945-1950. [CrossRef] 
24. Grollier, N.; Ansel, G.; Laot, C. Robust OFDM Modem for a Swarm of Underwater Autonomous Vehicles. In Proceedings of the OCEANS 2019, Marseille, France, 17-20 June 2019; pp. 1-6.

25. Lmai, S.; Bourré, A.; Laot, C.; Houcke, S. Advantages of pulse-shaping applied to OFDM systems over underwater acoustic channels. In Proceedings of the 2012 Oceans, Hampton Roads, VA, USA, 14-19 October 2012; pp. 1-7.

26. Gussen, C.M.G.; Diniz, P.S.R.; Campos, M.L.R.; Martins, W.A.; Costa, F.M.; Gois, J.N. A Survey of Underwater Wireless Communication Technologies. J. Commun. Inf. Syst. 2016, 31, 242-255. [CrossRef]

27. Gussen, C.M.G.; Laot, C.; Socheleau, F.X. Adaptive optimization of OFDM Underwater Acoustic Communication parameters based on on-board channel simulations. In Proceedings of the 6th International Conference \& Exhibition on Underwater Acoustics, Virtual Conference, Virtual, 21-24 June 2021.

28. Li, Y.; Cimini, L.J. Bounds on the interchannel interference of OFDM in time-varying impairments. IEEE Trans. Commun. 2001, 49, 401-404. [CrossRef]

29. Socheleau, F.-X.; Laot, C.; Passerieux, J.-M. Concise Derivation of Scattering Function from Channel Entropy Maximization. IEEE Trans. Commun. 2010, 58, 3098-3103. [CrossRef]

30. Socheleau, F.-X.; Laot, C.; Passerieux, J.-M. Parametric Replay-Based Simulation of Underwater Acoustic Communication Channels. IEEE J. Ocean. Eng. 2015, 40, 796-806. [CrossRef]

31. Socheleau, F.-X.; Laot, C.; Passerieux, J.-M. A Maximum Entropy Framework for Statistical Modeling of Underwater Acoustic Communication Channels. In Proceedings of the IEEE OCEANS'10 Conference, Sydney, Australia, 24-27 May 2010 ; pp. 1-7.

32. Chitre, M. A high-frequency warm shallow water acoustic communications channel model and measurements. J. Acoust. Soc. Am. 2007, 122, 2580-2586. [CrossRef]

33. Thorp, W.H. Deep-ocean sound attenuation in the sub-and low-kilocycle-per-second region. J. Acoust. Soc. Am. 1965, 38, 648-654. [CrossRef]

34. Van Walree, P.A.; Otnes, R.; Jenserud, T. The Watermark Manual and User's Guide, Version 1.0; FFI Rapport; Norwegian Defence Research Establishment (FFI): Kjeller, Norway, 2016.

35. van Walree, P.A.; Socheleau, F.-X.; Otnes, R.; Jenserud, T. The Watermark Benchmark for Underwater Acoustic Modulation Schemes. IEEE J. Ocean. Eng. 2017, 42, 1007-1018. [CrossRef] 